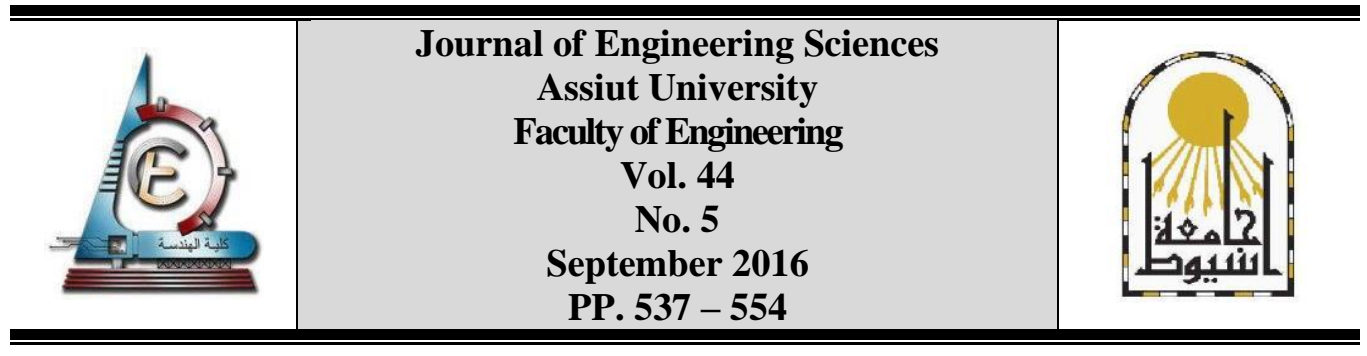

\title{
JOINT COMPRESSIVE SENSING FRAMEWORK FOR SPARSE DATA/CHANNEL ESTIMATION IN NON-ORTHOGONAL MULTICARRIER SCHEME
}

\author{
Mostafa Salah ${ }^{1}$, Osama A. Omer ${ }^{2}$ and Usama Sayed Mohamed ${ }^{*, 3}$ \\ ${ }^{l}$ Dept. of Electrical Engineering, Sohag University, Sohag, Egypt \\ ${ }^{2}$ Dept. of Electrical Engineering, Aswan University Aswan 81542, Egypt \\ ${ }^{3}$ Dept. of Electrical Engineering, Assiut University, Assiut, Egypt
}

Received 20 July 2016; Accepted 21 August 2016

\begin{abstract}
Many wireless channel behavior exhibits approximate sparse modeling in time domain, therefore compressive sensing (CS) approaches are applied for more accurate wireless channel estimation than traditional least squares approach. However, the CS approach is not applied for multicarrier data information recovery because the transmitted symbol can be sparse neither in time domain nor in frequency domain. In this paper, a new Sparse Frequency Division Multiplexing (SFDM) approach is suggested to generate sparse multicarrier mapping in frequency domain based on the huge combinatorial domain. The subcarriers will be mapped in sparse manner according to data stream for taking advantages of multicarrier modulation with lower number of subcarriers. The number of activated subcarriers is designed to achieve the same as Orthogonal Frequency-Division Multiplexing data rate under lower signal-to-noise ratio. The proposed approach exploits the double sparsity of data symbol in the frequency domain, and channel sparsity in the time domain. The same CS approach for both data recovery and adaptive channel estimation in a unified sparsely manner is used. The suggested framework can be used with any non-orthogonal waveform shaping and can work efficiently without any prior information about neither the channel sparsity order nor searching for the optimum pilot patterns.
\end{abstract}

Keywords: Compressive sensing; sparse channel estimation; super-resolution; non-orthogonal waveforms; sparse frequency division multiplexing (SFDM); combinatorial sparsifying.

\section{Introduction}

Multicarrier (MC) communication gives a solution for sever inter-symbol interference (ISI) in high data rates by dividing the high data rate into smaller sub-streams with lower rates which can be loaded on independent sub-carrier. The OFDM system is a special form of MC that has orthogonal sub-carriers which has been applied in many wireless standards. The orthogonality condition allows spectrum overlapping without interference between subcarriers, hence, more efficient spectrum usage. Also, it has simple generation and recovery via simple and fast FFT operation. Moreover, it has simple channel equalization as simple one complex multiplication

* Corresponding Author.

Email address: usama@aun.edu.eg 
per subcarrier in the frequency domain. On the other hand, the OFDM shortcomings are the high Peak-to-Average Power Ratio (PAPR), high Out-Off-Band (OOB) emission due to large side-lobes of rectangular window (large number of null guard subcarriers at spectrum edges, about $20 \%$ of the total allocated spectrum Bandwidth [1]), cyclic prefix (CP) overhead that adds nearly $25 \%$ of the symbol time, sensitivity to Doppler frequency shift, and Carrier Frequency Offset (CFO) [1-3]. Hence, the current direction is to get rid of the orthogonality limitations of the OFDM, and relay on a new non-orthogonal waveform design [4-8]. Good comparison among current competing multicarrier candidates is presented in [6]. It demonstrates the main rules that can be used for judging a communication technique. Moreover, as reported in [8], the employment of non-orthogonal pulses is optimum for minimizing ISI and ICI in double dispersive channels. However, sacrificing by the orthogonality results in better spectral usage at the cost of complex recovery.

Filter Bank Multicarrier (FBMC) finds increasing interest. It allows successive symbol overlap through data staggering on alternate in-phase and quadrature sub-channels. It exploits the staggered structures to achieve flexibility on filter design while maintaining the spectral efficiency of the unity time-bandwidth product. The spectral efficiency is due to working without $\mathrm{CP}$ and using smooth pulse shape, but at the cost of increased complexity due to the replacement of IFFT/FFT in OFDM with the filter banks and more complex equalization [9]. Our focus is to investigate that trend of incorporating non-orthogonal multicarrier schemes of arbitrary waveform other than rectangular waveform associated with OFDM system.

Orthogonality loss through windowing or shortening symbol interval (observation time) gives rise to spectral leakage between subcarriers due to boundary discontinuity. Therefore, it reduces spectral resolution resulting from Fourier analysis [10]. In resolving the problem, recalling sparse super-resolution tool can resolve the spectral lines of windowed symbol where Fourier tool fails. Super-resolution spectral estimation is introduced in literature, [11], [12], and continually discussed in recent research through spectral compressive sensing (SCS), [13]. It exploits prior knowledge of sparsely structure embedded in the signal for achieving super-resolution spectral estimation.

The original CS theory allows sparse signal recovery from under-sampled measurements under sparsity and bases incoherence constraints to enable signal compression while it is being acquired [14]. Moreover, it provides super-resolution ability of decomposing the signal into its sparsest components from proper over-complete dictionary. In this paper, the superresolution ability of sparsity based CS approaches will be exploited in resolving nonorthogonal MC modulation. CS approaches cannot be applied directly on the conventional MC modulated signals for data recovery because that signal doesn't satisfy sparsity constraint. Therefore, special sparsity mapping will be suggested for this purpose.

Exploiting enhanced recovery precision of sparsity approximation algorithm even under lower SNRs and reducing frequency spacing is the main motivation for the proposed sparsity mapping. Therefore, the achieved superior Bit-Error-Rate (BER) performance stems as a natural consequence of replacing conventional signal processing algorithms (such as filter bank and Fourier analysis) with the recently arising sparsity-based signal processing.

In this paper, sparsifying coding based on combination manner is suggested. Loading small number of subcarriers from the available subcarriers provides the frequency domain sparsity. By double sparsity of data (in the frequency domain) and channel (in the time domain), it is easy to incorporate the same hardware for both data recovery and channel 
estimation with two different dictionaries. Then, the advantages of the proposed sparsifying mapping can be summarized as follows: (1) Unifying data/ channel estimation in compressive sensing framework, (2) Exploiting super-resolution ability of CS-based approaches in resolving non-orthogonal MC systems, (3) Avoiding the problem of searching for optimum pilot pattern allocation, (4) unlike CoSaMP [25], the proposed system don't have the prior knowledge constraint of channel sparsity order (number of dominant paths of the channel) which is not available practically, and (5) Spectral efficiency resulting from minimizing training symbol rate and lower side lobes power concerned to non-orthogonal waveform. The rest of the paper is organized as follows. Section 2 overviews the application of CS on estimating channel sparsity. The followed sparse approximation tool is presented in Section 3. The proposed scheme along its complexity is introduced in Section 4 including combinatorial mapping, data/channel dictionaries and adaptive equalization. Then, the simulation results are introduced in Section 5. Finally, Section 6 concludes the paper.

\section{Compressive sensing sparse channel estimation}

Recently, CS approaches have given more attention in the field of system identification such as wireless channel estimation. Basically, many practical wireless channel behaviors can be modeled by impulse response having few and distributed (sparse) number of taps. This behavior becomes clearer in large BW signal, longer symbol interval and large number of antennas. Under channel sparsity, the application of recently proposed CS approaches introduces much better accurate channel estimation with aid of much less training pilots than traditional LS technique [16 - 24]. In [17], Matching Pursuit (MP) algorithm was applied in channel estimation of UWB system. Also, the work in [15] presents symbol detection along with channel estimation in UWB. While the nature of Ultra Wide Band (UWB) communications allows for double sparsity in time domain for both transmitted data information pulses besides channel impulse response [15], multicarrier (MC) systems cannot exhibit data sparsity neither in time domain nor in frequency domain. The proposed approach in [18] deals with the CS based channel estimation of multicarrier case in general. It compares the performance of three CS algorithms: Basis Pursuit (PB), Orthogonal Matching Pursuit (OMP) and Compressive Sampling Matching pursuit (CoSaMP). It is concerned in achieving spectral saving through reducing number of pilot symbols. Operation at OFDM with reduced speed of the analog-to-digital converter (ADC) on channel estimation part is introduced in [20].

Sparsity-based solutions can be classified into two main groups, namely, convex optimization and greedy algorithms [25]. Because the performance of the greedy methods is more influenced by coherence of the used measurement matrix, there is a problem of optimum pilot allocation [26]. Many studies are presented for minimizing mutual coherence of the measurement matrix and searching for optimum pilot pattern [25-32], which can be avoided in the suggested framework in this paper. While traditional OFDM channel estimation depends on the interpolation of equally spaced pilot subcarriers, equally spaced pattern may not be the optimum pattern in respect to CS approaches. The problem of optimum pilot pattern allocation takes a lot of study. The optimal pilot pattern was found to be the randomly generated pattern. But, due to difficulty of random patterns support, there are many algorithms for searching for the optimum deterministic patterns [27, 28, 31-33]. 


\section{Sparse iterative covariance-based estimation (SPICE)}

SPICE is a robust high resolution sparse estimator. It is our choice to be applied on the problem of sparse data/channel estimation. It provides sparse parameter estimation for the following traditional linear model:

$$
\mathrm{y}=\mathrm{Bx}+\mathrm{e}
$$

Where, $y \in C^{M}$ is observation vector, $B \triangleq\left(b_{1} b_{2} \ldots \ldots b_{N}\right) \in C^{M \times N}$ is the regression matrix (measurement dictionary), $x \in \mathrm{C}^{\mathrm{N}}$ denotes the unknown sparse parameter vector with the number of measurements $M$ can be less than number of regression vectors, $N$, and $e$ is unknown additive noise. It is required to choose such dictionary (domain) that allowing estimation of the sparse representation $\mathrm{x}$ for the observation signal $y$. The prior-knowledge of sparsity presented in the parameter vector can be exploited in the solution of the following constrained LS problem where the sparsity condition is included through the $\mathrm{l}_{1}$ - norm.[34]

$$
\min _{\left\{x_{k}\right\}}\left\|y-\sum_{k=1}^{N} b_{k} x_{k}\right\|^{2} \quad \text { s.t. }\|x\|_{1} \triangleq \sum_{k=1}^{N}\left|x_{k}\right| \leq \mu
$$

Where $\|\cdot\|_{1}$ stands for $l_{1}$ - norm, and $\mu$ is certain threshold specified by the user. That can be interrupted as a weighted, hyperparameter-free square-root LASSO.[40]

$$
\arg \min _{\mathrm{x}}\|\mathrm{y}-\mathrm{Bx}\|_{2}+\|\mathrm{Dx}\|_{1}
$$

Where $\mathrm{D}=\operatorname{diag}\left(\sqrt{\frac{\left\|\mathrm{b}_{1}\right\|^{2}}{\mathrm{M}}} \ldots \ldots \ldots \ldots \ldots . \sqrt{\frac{\left\|\mathrm{b}_{\mathrm{N}}\right\|^{2}}{\mathrm{M}}}\right)$. The main advantages of SPICE can be summarized as follows; 1) it doesn't need the knowledge of sparsity order (number of significant elements or number of channel taps w.r.t channel model), 2) it operates in iterative manner with global convergence (convexity), 3 ) it is adapted for complex-valued data and parameters as simply as real-value, 4$)$ and it is more accurate than its competitive algorithm BP [38]. Hence, it can be deduced that SPICE can be more accurate than any variants of MP algorithm which were widely used in previous CS-based channel estimation algorithms. Iterations loops are performed between covariance matrix computation and power estimate update with constant weights. Complete derivation, explanation, and suggested fast implementations of SPICE can be found in [34-40]. Moreover, its MATLAB implementation is published on the author's page [39, 40].

\section{The Proposed unified sparse data/channel estimation framework}

It is a new MC scheme operating entirely in CS domain through sparsely mapping subcarriers for allowing the sparse CS approaches to be applied on sparse subcarriers data recovery in the frequency domain as well as sparse channel estimation in the time domain. Figure 1 shows the block diagram of the proposed "Unified sparse data/channel estimation framework". In the following Subsections, the proposed framework will be explained in details.

\subsection{Combinatorial sparse frequency division multiplexing (SFDM)}

MC is robust against ISI but at the cost of its extremely higher PAPR. Sparsity based super-resolution approaches cannot be exploited directly for data recovery in Multicarrier modulation neither in the time domain nor in the frequency domain. Intermediate case between the two types is suggested by reusing the available large number of subcarriers in 
such different manner that can result in better sparse frequency representation while regarding the achieved bit rate of traditional $\mathrm{MC}$ systems. By loading only $\mathrm{N}_{\mathrm{S}}$ active subcarriers taken out from $\mathrm{N}_{\mathrm{T}}$ available subcarriers $\mathrm{N}_{\mathrm{S}}<\mathrm{N}_{\mathrm{T}}$. The information doesn't reside in active or passive subcarriers, but it can be recovered from the subcarriers distribution between passive and active or their combination. Figure 2 illustrates the difference between normal denes (top) against the suggested sparse (bottom) multi-carrier.

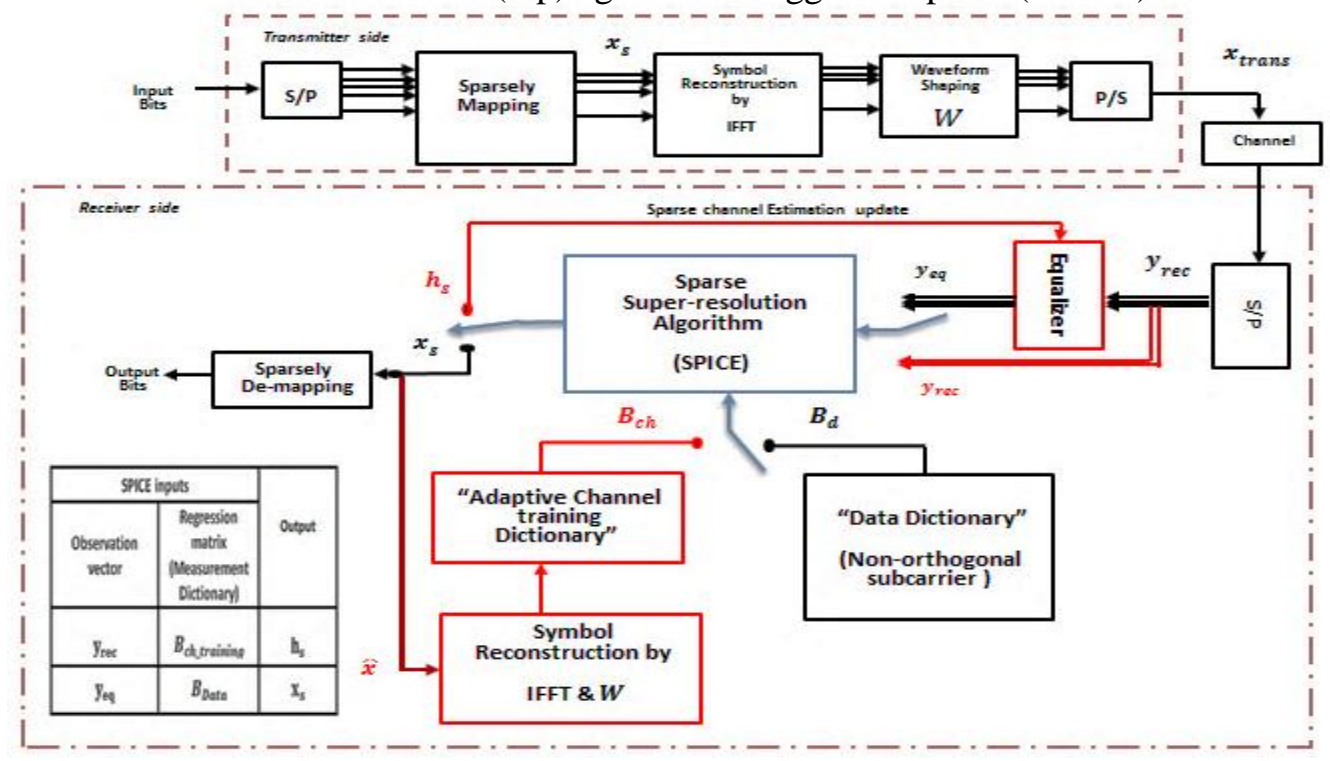

Fig. 1. Block diagram of the proposed "Unified sparse data/channel estimation framework"
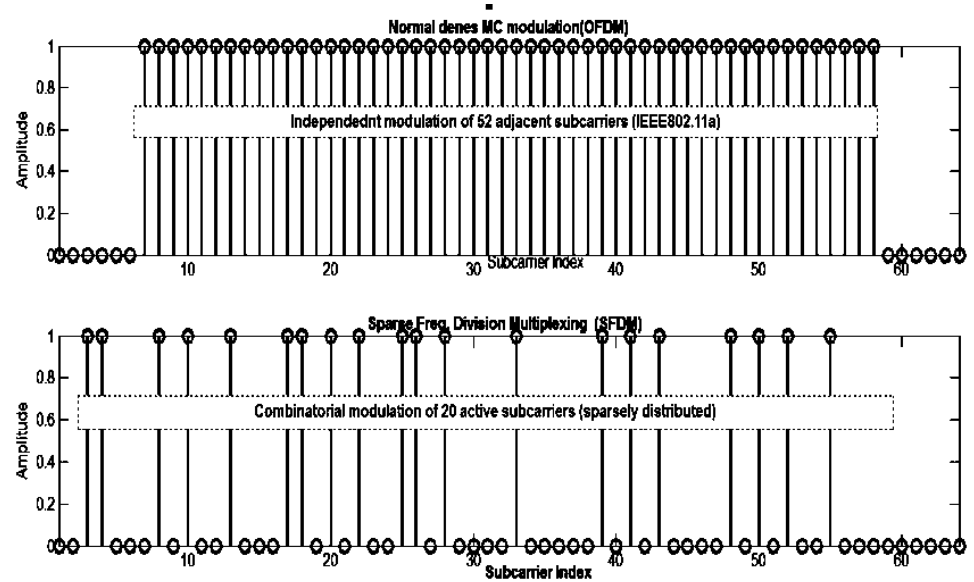

Fig. 2. Denes against sparse MC modulation

It is known that the combination is a way of selecting (activating) $N_{S}$ subcarriers out of a collection $N_{T}$ (total available subcarriers in the frequency band) while the order of selection is not important. The number of possible combinations can be given as follows:

$$
\mathrm{C}=\left(\begin{array}{c}
N_{T} \\
N_{S}
\end{array}\right)=\frac{N_{T} !}{N_{S} !\left(N_{T}-N_{S}\right)}
$$

Where ! stands for the factorial.

That recalls combinatorial number system which can provide rapid unique representation for each possible combination arranged in lexicographic order [44-46]. 
Combinations are assumed to be ordered in a predefined (or assumed) order that can be computed easily, where the mapping process from the order index to the combination pattern and its reverse belong to combinatorial number algorithms.

For each resultant combination there is $\mathrm{M}$ possible modulation state $(\mathrm{M}=2$ for BPSK \& $\mathrm{M}=4$ for QPSK) for each subcarrier. Then the overall possible combinations of $\mathrm{N}_{S}$ subcarriers out of $\mathrm{N}_{\mathrm{T}}$ is equal $\mathrm{M}^{\mathrm{N}_{\mathrm{S}}} *\left(\begin{array}{c}\mathrm{N}_{\mathrm{T}} \\ \mathrm{N}_{\mathrm{S}}\end{array}\right)$. The equivalent decoded bits can be computed by taking $\log _{2}$ of the possible combinations as follows:

$$
\mathrm{d}=\log _{2}\left\{\mathrm{M}^{\mathrm{N}_{\mathrm{s}}} *\left(\begin{array}{c}
\mathrm{N}_{\mathrm{T}} \\
\mathrm{N}_{\mathrm{S}}
\end{array}\right)\right\} \quad \text { bits }
$$

Each data block consisting of " $d$ " bits can be seen as the combination address (position index) for a certain combination. In the transmitter side, sparsity mapping is a process of picking the combination corresponding to the given combination index. The reverse process (sparsity demapping) is performed in the receiver side. For example, assume the system with 64 subcarriers, $\mathrm{N}_{\mathrm{T}}=60$ (let 4 as guard subcarriers at edges), and the active (selected) subcarriers $\mathrm{N}_{\mathrm{S}}$ is changed from 1 to 20 . Then, the coded bits against $\mathrm{N}_{S}$ will be changed as shown in Fig. 3.

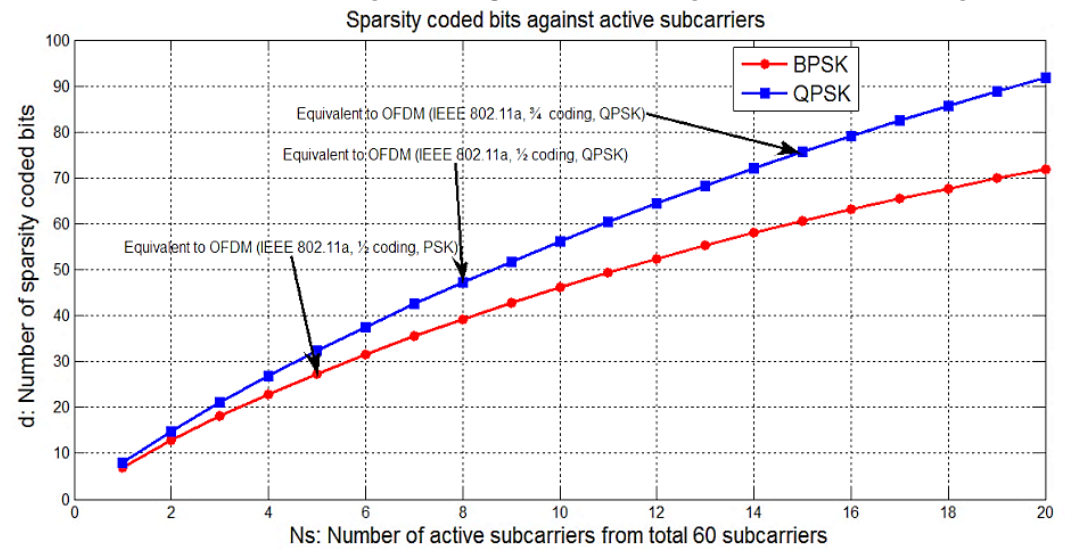

Fig. 3. combination coded bits against active subcarriers

From Figure 3, it is clear that the bit rate of the 64-subcarrier of the OFDM system (IEEE, 802.11, QPSK modulated, 1/2 channel coding: 48 active data subcarriers +4 active pilots +12 Null), can be achieved using only 8 active, sparsly scattered with QPSK modulated subcarriers from the available 64 frequency bins instead of activating 52 subcarriers simultaneously. The main drawback of this approach is that an error of any subcarriers will cause an error in all symbol block. But, the robust sparse recovery technique guarantees acceptable recovery levels.

\subsection{Symbol generation and waveform shaping (transmitter system)}

In the proposed system, instead of transmitting the data using all subcarriers, the data are transmitted using sparse number of subcarriers. As shown in Fig. 1, the input data stream of length " $\mathrm{d}$ " bits will be converted into parallel form and mapped into coded combination corresponds to that bit pattern. The resultant sparse coding $\left(\mathrm{x}_{\mathrm{S}}\right)$ will be considered as sparse frequency representation of multicarrier system. The IFFT of $\left(\mathrm{x}_{\mathrm{S}}\right)$, will result in the equivalent time domain symbol. To that point the time domain signal represents sparse orthogonal modulated subcarriers. By applying arbitrary waveform 
shaping (W) such as Hamming, Hanning, Kaiser, Gaussian or any other known windows [9], the symbol becomes non-orthogonal modulated subcarriers.

Without loss of generality, the exponentially damped waveform shaping [47] (shown in Fig. 4) will be considered. The motivation of using the Exponentially damped (ED) window is that; (1) the damping window has highly attenuated end, hence, the symbol is terminated with lower energy with respect to its start, (2) Any partially overlap (at maximum, about $25 \%$ of symbol interval, corresponding to maximum channel spread) between consecutive symbols, will be considered an acceptable interference, and (3) the feature of the exponentially damped window allows transmission of consecutive symbols without Guard Interval (GI) or cyclic prefix (CP). Figure 5 shows the comparison between the ISI in case of original OFDM without CP, and the ISI in the damped case without GI. From this figure, it can be shown that using damped window has lower ISI without using GI compared to the conventional OFDM without using CP.

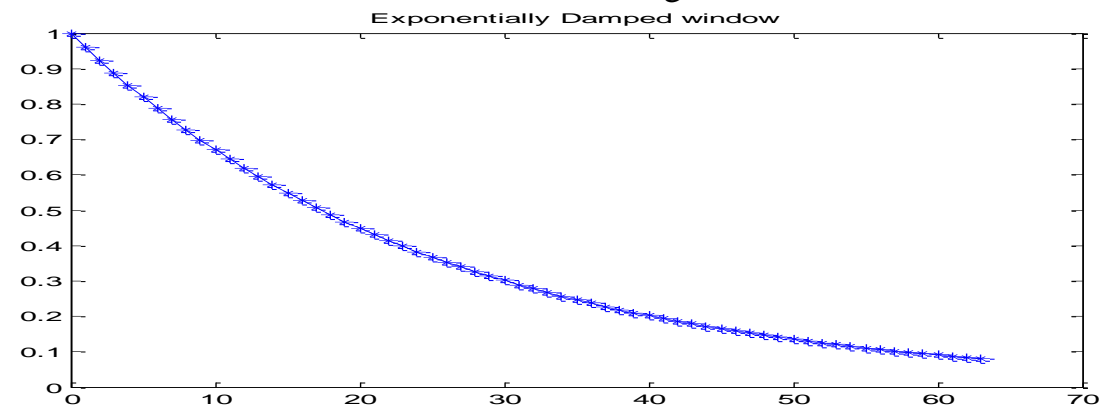

Fig. 4. exponentially damping waveform
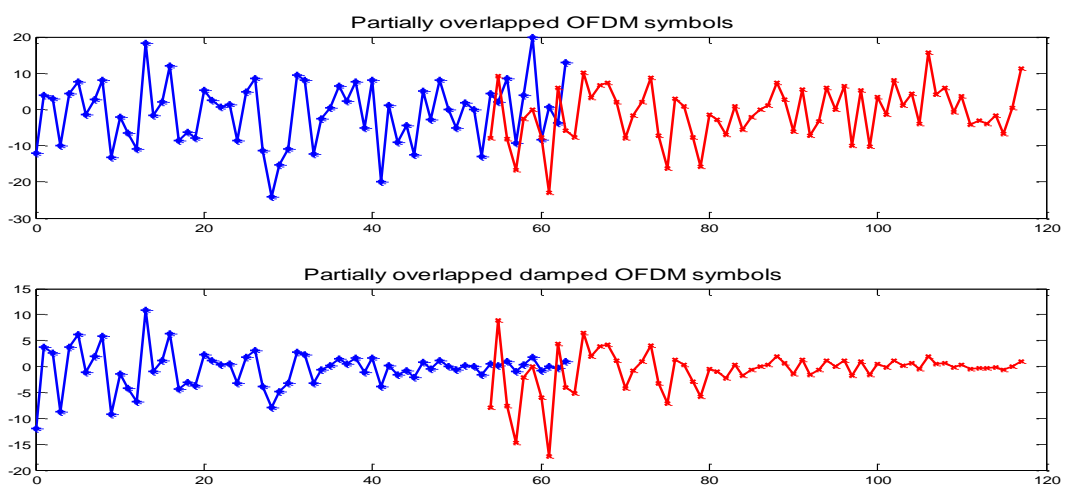

Fig. 5. ISI effect in both un-damped OFDM and damped case

\subsection{Channel measurement matrix}

The received signal is the sum of all multipath copies (scaled and delayed) of the transmitted signal $x_{\text {trans. }}$. In training based channel estimation, the transmitted training symbol $x_{\text {trans_training }}$ is known in the receiver. The received symbol $y_{\text {rec }}$ is resulting from convolving $\mathrm{x}_{\text {trans_training }}$ with the unknown sparse channel impulse response $(\mathrm{h})$.

$$
\begin{aligned}
y_{\text {rec }} & =\operatorname{conv}\left(x_{\text {trans_training }}, h\right)+e \\
& =B_{\text {ch_training }} \cdot \boldsymbol{h}_{\boldsymbol{s}}+e
\end{aligned}
$$

Where, $h_{s}=\left(h_{0} h_{1} h_{2} \ldots \ldots \ldots . h_{L-1}\right)^{T}$ represents $L$ taps channel impulse response and $\mathrm{e}$ is the additive noise error. Eq. (6) can be reformed in matrix form [24, 25, 29] by 


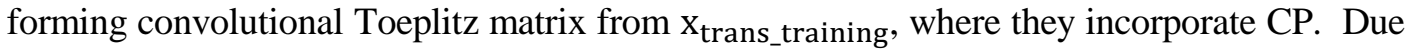
to using the smoothly ended (damped) non-orthogonal waveforms and assume that the maximum channel spread is no longer than $25 \%$ of symbol length, ISI between consecutive symbols can be neglected. Therefore, the convolution matrix can be written as follows:

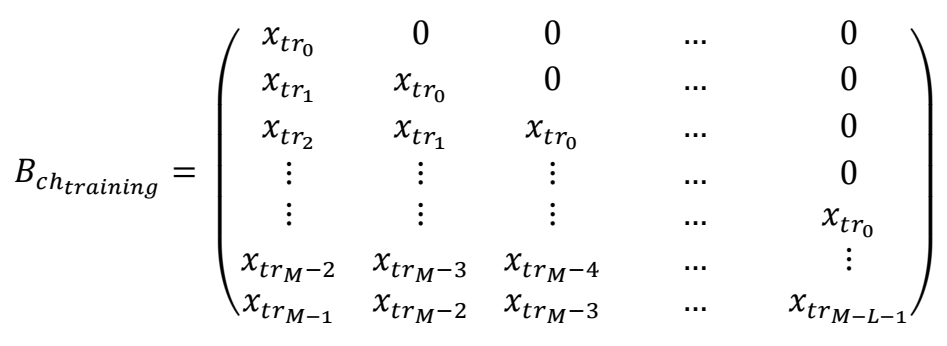

\subsection{Data measurement matrix $\left(\boldsymbol{B}_{\boldsymbol{d}}\right)$}

The data measurement matrix is windowed Fourier bases (equally spaced) subcarriers in the allocated bandwidth (BW). Let, the total numbers of possible subcarriers $\mathrm{N}_{\mathrm{T}}$ equals the number of columns of the measurement matrix $B_{d} \in C^{M \times N_{T}}$, and $y_{e q} \in C^{M}$ represents the equalized symbol which approximates the transmitted symbol $x_{\text {trans }}$. Let $\mathrm{x}_{\mathrm{S}} \in \mathrm{C}^{\mathrm{N}_{\mathrm{T}}}$ is the required sparsely coded subcarriers vector. i.e.,

$$
\begin{gathered}
y_{e q} \approx x_{\text {trans }}=B_{d} x_{s}+e \\
B_{d}=F \odot W
\end{gathered}
$$

Where

$$
\begin{gathered}
F=\left(\begin{array}{ccccc}
1 & 1 & 1 & \ldots \ldots \ldots & 1 \\
1 & e^{j \omega_{1}} & e^{j \omega_{2}} & \ldots \ldots \ldots & e^{j \omega_{N-1}} \\
1 & e^{j 2 \omega_{1}} & e^{j 2 \omega_{2}} & \ldots \ldots \ldots & e^{j 2 \omega_{N-1}} \\
1 & e^{j 3 \omega_{1}} & e^{j 3 \omega_{2}} & \ldots \ldots \ldots & e^{j 3 \omega_{N-1}} \\
1 & e^{j 4 \omega_{1}} & e^{j 4 \omega_{2}} & \ldots \ldots \ldots & e^{j 4 \omega_{N-1}} \\
\vdots & \vdots & \vdots & \vdots & \vdots \\
1 & e^{j M \omega_{1}} & e^{j M \omega_{2}} & \ldots \ldots \ldots & e^{j M \omega_{N-1}}
\end{array}\right) \\
\& W=\left(\begin{array}{cccc}
w_{0} & w_{0} & \ldots & w_{0} \\
w_{1} & w_{1} & \ldots & w_{1} \\
\vdots & \ddots & \vdots & \vdots \\
w_{M-1} & w_{M-1} & \ldots & w_{M-1}
\end{array}\right) \& x_{s}=\left(\begin{array}{c}
x_{0} \\
x_{1} \\
x_{2} \\
\vdots \\
x_{N-1}
\end{array}\right) \& y_{r e c}=\left(\begin{array}{c}
y_{r e c_{0}} \\
y_{r e c_{1}} \\
y_{\text {rec }} \\
\vdots \\
y_{r e c_{M-1}}
\end{array}\right)
\end{gathered}
$$

Where $\mathrm{F}$ the Fourier matrix with each column represents subcarrier and $\mathrm{W}$ is the used waveform. Note that $(\odot)$ represents a simple element-by-element multiplication. It is the role of the super-resolution sparse approach to estimate the sparse channel $h_{s}$ from Eq. 6 , and the sparsely coded data $\mathrm{x}_{\mathrm{s}}$ from Eq. 7 .

\subsection{Adaptive equalization}

As in training based channel estimation scheme, the training symbols are time multiplexed periodically with data symbols by certain rate to allow following channel variation at receiver side. By incorporating adaptive equalization, the rate of training symbol transmission overhead can be reduced, hence increasing data rate. As soon as the sparse coded symbol $\left(\hat{\mathrm{x}}_{\mathrm{S}}\right)$ being recovered from $\mathrm{y}_{\mathrm{eq}}$ by the aid of data dictionary, it can be reused for playing the role of training symbol, by taking IFFT transform and waveform shaping for reproducing the time 
symbol as generated in transmitter side, hence, reconstructing training matrix from it. By that, the needed rate of training symbols can be reduced, because each recovered symbol will be exploited as training symbol for next symbol equalization. Original agreed training symbol can be requested by receiver in case of remarking increased error rate at receiver side. The steps of the proposed approach can be shown in the following algorithm:

\subsection{The Computational complexity of the proposed approach}

In the proposed approach, the transmitter complexity is similar to the OFDM system; however, the receiving operation is more complex. The receiver computational complexity is related to the applied CS algorithm. In this paper, the SPICE is used for sparsity approximation; hence, it imposes its complexity on the proposed scheme.

The SPICE complexity is of the order $\mathrm{O}(\mathrm{N} 2)$ per iteration which is corresponding to the DFT complexity. The number of iterations is adjusted according to the SNR of the received signal. More efficient implementation of the SPICE based on the GohbergSemencul (G-S) factorization is presented in [36] for array processing which may be exploited in multi-input-multi-output (MIMO) extension.

Although sparsity approximation algorithms (such as SPICE and OMP) are more complex than FFT complexity employed in the OFDM transmission/recovery, it is not fair to be compared with the OFDM system for the following reasons:-

1) Fourier analysis is not succeeded under non-orthogonal schemes.

2) Fourier analysis does not utilize the benefits resulting from the signal embedded sparsity.

3) Sparsity approximation algorithms can provide better BER performance along with the proposed sparsity mapping (it will be described later).

4) Also, under the hardware realization, the proposed scheme doesn't require independent implementation for channel estimation and data recovery modules as a direct consequence of double sparsity employment. The same hardware module can be utilized for both data detection /channel estimation.

5) Proposed CS-based scheme doesn't employ any channel coding and decoding overhead/complexity (such as convolutional coding or LDPC coding) without performance degradation. Moreover, it achieves better performance than the codedOFDM system under lower SNRs.

Hence, the fair comparison may be performed with the non-orthogonal MC schemes such as the FBMC system.

In general, the optimum CS algorithm, the computational complexity, the recovery success rate, the required number of iterations and the operating SNR is considered an open problem for further research in achieving the optimum sparsity approximation performance [41- 43]. 
Pseudo Code for the Proposed Algorithm

1. Apply SPICE

Estimating channel $\mathrm{h}_{\mathrm{s}}^{(\mathrm{i})}$ from received symbol $\mathrm{y}_{\mathrm{rec}}^{(\mathrm{i})}$ for symbol "i" with aid of $\mathrm{B}_{\text {ch_training. }}$.

2. Channel Equalization

Getting equalized symbol $\mathrm{y}_{\mathrm{eq}}^{(\mathrm{i}+1)}$ from $\mathrm{y}_{\text {rec }}^{(\mathrm{i}+1)}$ depending on $\mathrm{h}_{\mathrm{s}}^{(\mathrm{i})}$.

3. Apply SPICE

Estimating sparsely coded data $\mathrm{x}_{\mathrm{s}}^{(\mathrm{i}+1)}$ from $\mathrm{y}_{\mathrm{eq}}^{(\mathrm{i}+1)}$ with the aid of $\mathrm{B}_{\mathrm{d}}$.

4. Bit Stream Recovery

De-mapping $\mathrm{x}_{\mathrm{s}}^{(\mathrm{i}+1)}$ to produce original bit stream.

5. Adaptive Cannel Estimation

- $\mathrm{x}_{\mathrm{s}}^{(\mathrm{i}+1)} \rightarrow \mathrm{x}_{\mathrm{pilots}}^{(\mathrm{i}+1)}$

//reconsider sparse coded subcarriers $\mathrm{x}_{\mathrm{s}}^{(\mathrm{i}+1)}$ as the recent pilot symbol pattern.

- Reconstructing $\mathrm{x}_{\text {trans }}^{(\mathrm{i}+1)}$ from $\mathrm{x}_{\mathrm{s}}^{(\mathrm{i}+1)}$, by taking IFFT \& waveform shaping

- $\mathrm{x}_{\text {trans }}^{(\mathrm{i}+1)} \rightarrow \mathrm{x}_{\text {trans-training }}^{(\mathrm{i}+1)}$

//Reconstruct transmitted training signal $\mathrm{x}_{\text {trans }}^{(\mathrm{i}+1)}$ from the assumed pilot symbol $\mathrm{x}_{\mathrm{s}}^{(\mathrm{i}+1)}$

6. Reconstructing new $\mathrm{B}_{\text {ch_training }}$ from $\mathrm{x}_{\text {trans-training }}^{(\mathrm{i}+1)}$

7. Increment $\mathrm{i}$

8. Return to step 1

\section{Simulation results}

This section demonstrates the previously supposed advantages of the proposed scheme. First, the data/channel recovery using the proposed framework will be shown for nonorthogonal waveform. Then, sparsity adaptation versus channel coding strategy will be confirmed. The next point is the problem of pilot pattern coherence and how to avoid its effects. The effect of sparsely coding on symbol energy and PAPR will be shown based on rectangular window used for normal OFDM. At the end, spectrum efficiency will be compared to conventional OFDM.

\subsection{Channel estimation}

The data symbols will be formed in EDS (Exponentially damped Sinusoidal) model in transmitter side at single damping factor (set to 0.035) with sparsely coded subcarriers. The modulation type is QPSK for active subcarri537537ers $\left(\mathrm{N}_{\mathrm{S}}=20, \mathrm{~N}_{\mathrm{T}}=60\right)$ under $\mathrm{SNR}=20 \mathrm{~dB}$. As shown in Fig. 6a, it presents the received and equalized symbols due to the supposed and estimated channels in Fig. 6b.

Analysis of the channel estimation error is out of scope of the proposed scheme because it is not limited to certain sparsity approximation algorithm. It shares the same recovery precision of the already applied algorithm under random selected pilot's patterns. The novelty of the proposed originates from allowing the reuse of the same sparsity approximation algorithm in estimating the channel sparsity to reapply the data recovery after the sparsity mapping. 


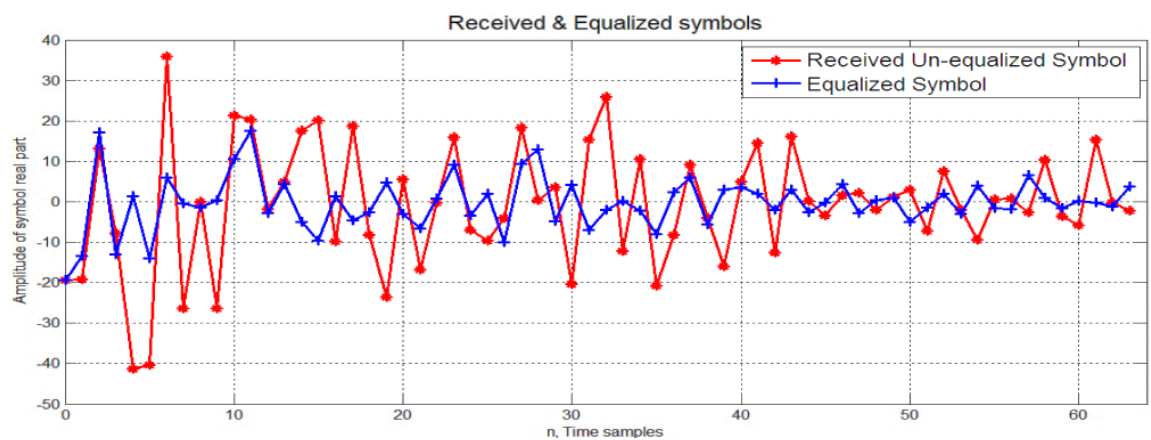

(a)

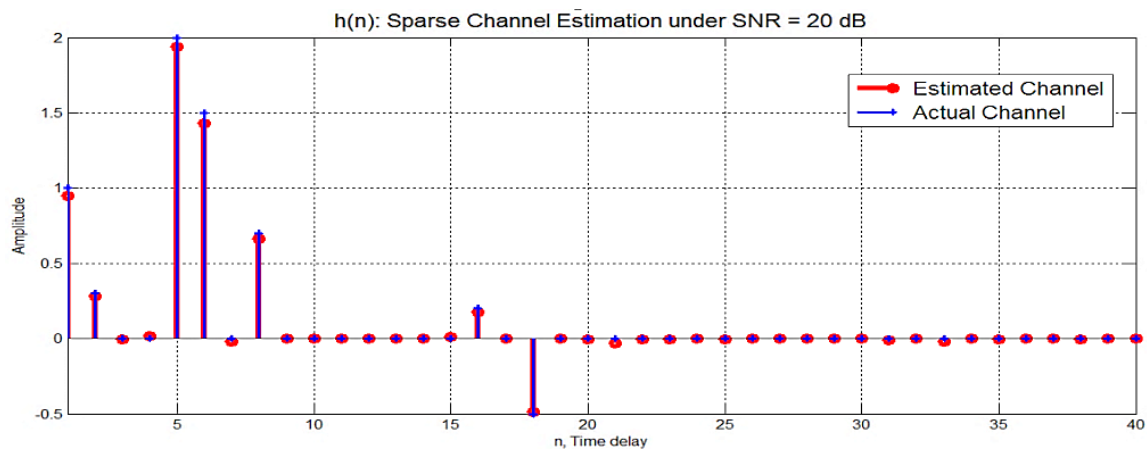

(b)

Fig. 6. (a) Received and equalized symbols, (b) original and estimated channel impulse response

\subsection{Sparsity adaptation versus channel coding}

The purpose of adding channel coding redundancy is to correct possible errors resulting at low SNR levels. Increasing coding redundancy increases signal immunity at cost of reduced data bits. With respect to our proposed SFDM and based on the observation that under given SNR, the probability of correct sparse data recovery varies depending on sparsity level $\left(\mathrm{S}=\mathrm{N}_{\mathrm{S}} / \mathrm{N}_{\mathrm{T}}\right)$. By decreasing " $\mathrm{S}$ ", signal sparsity order was enhanced; hence better recovery under lower SNR levels. New data protection strategy can be defined as varying the sparsity level instead of traditional channel coding based on redundancy insertion. (Note that increasing $\mathrm{S}$ beyond certain level is not allowed because it destroys sparsity constraint and degrades the overall performance). The following table provides analogy between channel coding rate of conventional OFDM system and the sparsity level adaptation suggested in our sparse multicarrier modulation (SFDM) system. Figure 7, represents acceptable data recovery under sparsity adaptation $\left(\mathrm{N}_{\mathrm{S}}: 16-3\right)$ for various $\mathrm{SNRs}$ (from $20-0 \mathrm{~dB}$ ).

It is worth mentioning that the simulations (in Fig. 7) was performed on ED window which exhibits severe attenuation and therefore, lower energy per symbol w.r.t other waveforms such as the Hanning waveform. So, the expected recovery performance for other waveforms is better under the same SNRs.

In [42], exact recovery performance of the sparsity approximation algorithms is sensitive to the signal sparsity order. It is observed in Fig. 7, where the accepted sparsity recovery of high/low sparsity order(S) is achieved along with corresponding high/low SNRs. As shown in Fig.7.d, it has only acceptable recovery when $\mathrm{N}_{S}=3$ active subcarriers from $\mathrm{N}_{\mathrm{T}}=60$ total subcarriers under extremely low SNR $0 \mathrm{~dB}$. The increased noise power appears in 
increasing the estimated power (noise floor) at inactive (passive) subcarriers. The correct active subcarriers still have the largest power levels and can be easily distinguished from the passive subcarriers. The choice of the loaded sparsity level $\left(S=N_{S} / N_{T}\right)$ corresponding to operating SNR is very important for achieving accepted recovery level.
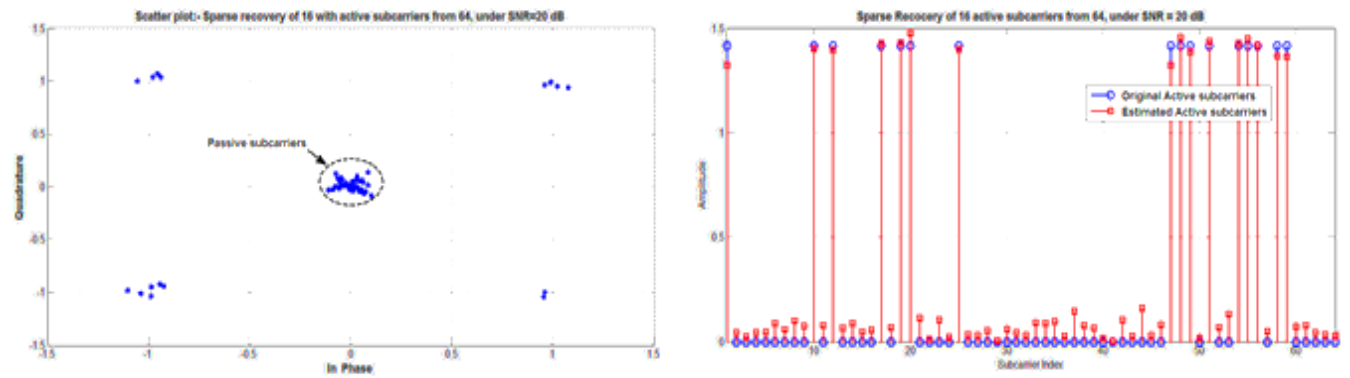

(a) $\mathrm{S}=\frac{\mathrm{N}_{\mathrm{S}}}{\mathrm{N}_{\mathrm{T}}}=\frac{16}{64}, S N R=20 \mathrm{~dB}$

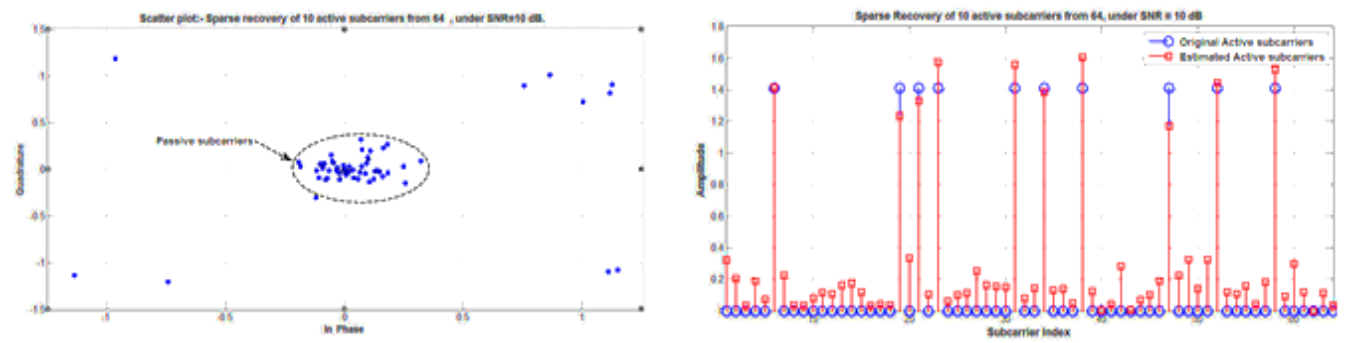

(b) $\mathrm{S}=\frac{\mathrm{N}_{\mathrm{S}}}{\mathrm{N}_{\mathrm{T}}}=\frac{10}{64}, \mathrm{SNR}=10 \mathrm{~dB}$
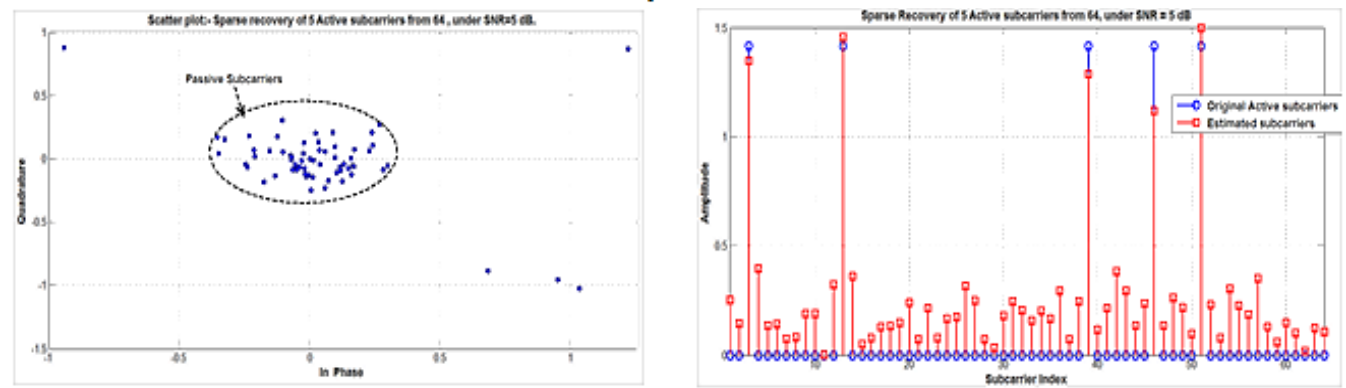

(c) $\mathrm{S}=\frac{\mathrm{N}_{\mathrm{S}}}{\mathrm{N}_{\mathrm{T}}}=\frac{5}{64}, \mathrm{SNR}=5 \mathrm{~dB}$
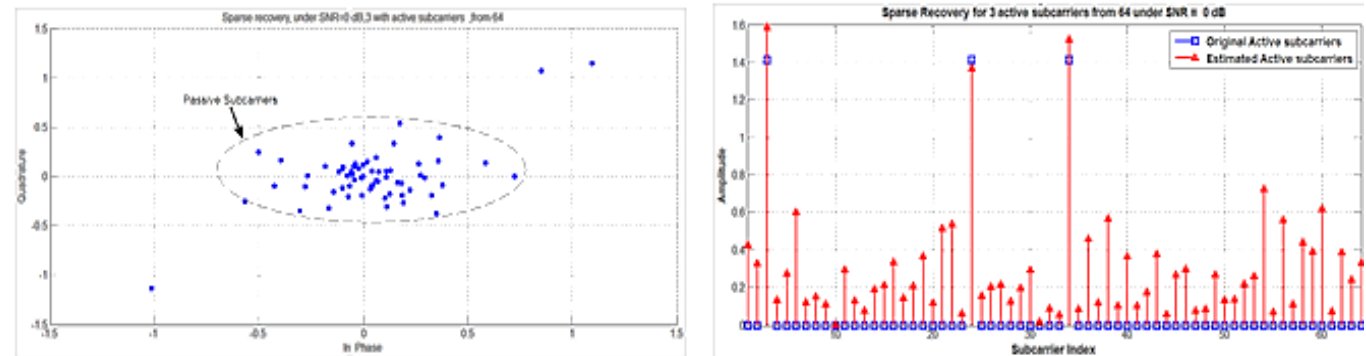

(d) $\mathrm{S}=\frac{\mathrm{N}_{\mathrm{S}}}{\mathrm{N}_{\mathrm{T}}}=\frac{16}{64}, S N R=20 \mathrm{~dB}$

Fig. 7. Sparsity adaptation strategy according to operating SNR 
Usama S. Mohamed et al., Joint compressive sensing framework for sparse data/channel

Table 1.

Sparsity adaptation versus channel coding

\begin{tabular}{|c|c|c|c|c|c|}
\hline \multirow{2}{*}{ 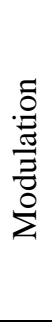 } & \multicolumn{3}{|c|}{$\begin{array}{c}\text { IEEE } 802.11 \mathrm{a}[48] \\
\text { (48 active data subcarriers }+4 \text { active pilots }+ \\
12 \text { Null })\end{array}$} & \multicolumn{2}{|c|}{$\begin{array}{c}\text { Proposed SFDM } \\
\text { (Sparse Frequency Division Multiplexing) }\end{array}$} \\
\hline & $\begin{array}{l}\text { Coding rate } \\
\text { (R) }\end{array}$ & $\begin{array}{l}\text { Coded bits per } \\
\text { subcarrier }\end{array}$ & $\begin{array}{l}\text { Data bits } \\
\text { per OFDM } \\
\text { symbol }\end{array}$ & $\begin{array}{c}\text { Sparsity level } \\
\mathrm{S}=\mathrm{N}_{\mathrm{S}} / \mathrm{N}_{\mathrm{T}} \\
\quad \text { where }\end{array}$ & $\begin{array}{l}\text { Data bits per } \\
\text { SFDM symbol } \\
\quad(\text { eq. 3) }\end{array}$ \\
\hline \multirow{3}{*}{$\frac{v}{\tilde{n}}$} & $1 / 2$ & 1 & 24 & $5 / 60$ & 27 \\
\hline & $3 / 4$ & 1 & 36 & $8 / 60$ & 39 \\
\hline & $\begin{array}{c}\text { without } \\
\text { channel coding }\end{array}$ & 1 & 48 & $11 / 60$ & 49 \\
\hline \multirow{3}{*}{$\begin{array}{l}\frac{1}{\tilde{2}} \\
\frac{2}{2}\end{array}$} & $1 / 2$ & 2 & 48 & $8 / 60$ & 47 \\
\hline & $3 / 4$ & 2 & 72 & $15 / 60$ & 75 \\
\hline & $\begin{array}{c}\text { without } \\
\text { channel coding }\end{array}$ & 2 & 96 & $20 / 60$ & 91 \\
\hline
\end{tabular}

As shown in Fig. 8, for SNR greater than $3 \mathrm{~dB}$, the proposed SFDM drastically reduces the Bit-Error-Rate (BER) compared to the conventional OFDM. In this figure, the coding rate is adjusted to $1 / 2$ for conventional OFDM and the sparsity level, while in the proposed SFDM the coding rate is adjusted to $8 / 60$.

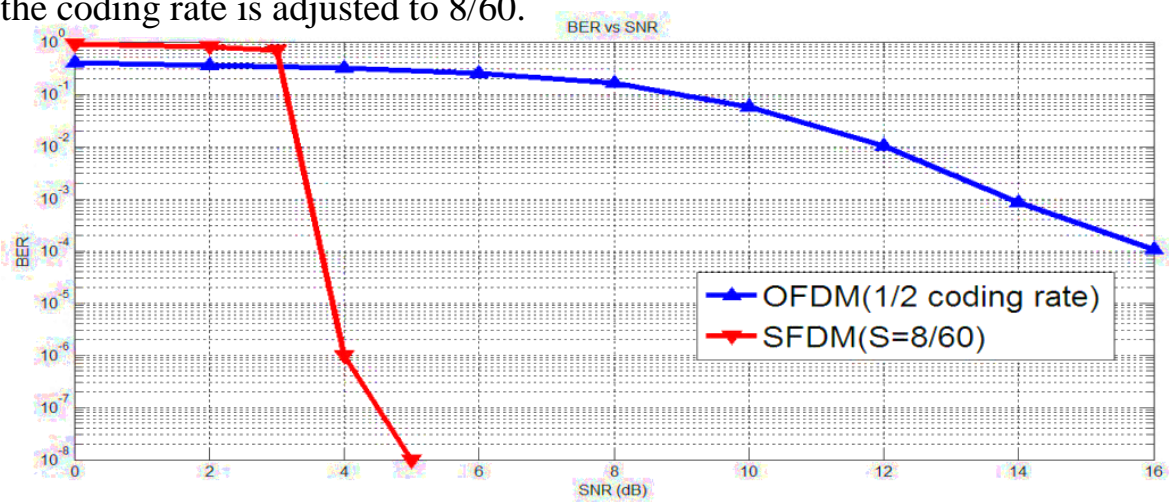

Fig. 8. BER comparison versus SNR

One observation can be emphasized from Fig.7 and 8, that each sparsity level has corresponding SNR for accepted recovery success rate. Sudden BER reduction w.r.t the proposed SFDM scheme occurs at SNR corresponding to the signal sparsity order. Justification of sudden BER reduction at extremely lower SNR, than required SNR for conventional coded OFDM, is stemming from the inspired robust performance of the sparsity approximation algorithms.

\subsection{Avoiding searching for optimum pilot pattern}

Sparse subcarriers can be considered as randomly generated according to the sparsely coded information bits which usually exhibits random style. Due to the double role played by the transmitted symbol, (first as data symbol, second as random pilot symbol after data recovery for updating channel state information), it has equivalently high pilot symbol rate with random pilot pattern. That randomness is the preferred pilot pattern with respect to the CS based estimators. 
For the worst conditions of the pilot pattern consisting of high coherence (that degrades sparsity approximation performance) components (such as consecutive subcarriers), the symbol can be dropped from updating channel estimation stage only, while the data can be recovered as shown in Fig. 9. This figure proves the case of acceptable recovery even for consecutive subcarriers.

\subsection{Reducing Symbol Energy and PAPR upper bound}

Due to the summation of large number of subcarriers in MC modulation, there is large probability for summing in such moment constructively giving rise to too large peeks w.r.t its average [49]. In that section, the effect of sparsely mapped subcarriers on both energy and PAPR will be demonstrated under the same waveform shape. To make fair comparison, normal OFDM signal is compared against SFDM under the same rectangular window as shown in Fig. 10a. Sparsely coding subcarriers (with active 8 subcarriers) lead to the predicted reduced symbol energy of about 0.25 ; while normal OFDM (densely modulated subcarriers) produces symbol energy of about 1.625. Energy reduction per the same transmitted bit rate represents a critical point for mobile battery based systems. Improved energy efficiency stems from the fact that the proposed SFDM activates lower number of sinusoidal subcarriers in symbol creation and the information was conveyed through pattern distribution of the subcarriers between active/passive states. On the other hand, without applying any PAPR reduction technique, Fig.10b, demonstrates the reduced PAPR for sparsely coding subcarriers.

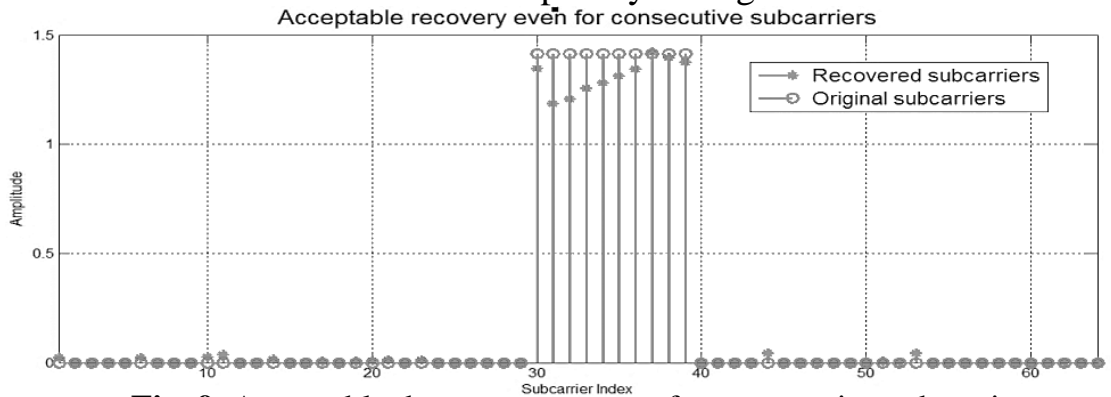

Fig. 9. Acceptable data recovery even for consecutive subcarriers

\section{Conclusion}

In this paper, a combinatorial multicarrier sparsifying (mapping) formulation in frequency domain was presented. Activating smaller number of subcarriers from the whole available (according to combinatorial mapping) gives rise to frequency domain sparsity. The double sparsity of the formulated sparse data model in frequency domain besides the already known channel sparsity in time domain, give us the chance for applying the same sparsity based super-resolution algorithm for jointly recovering data and channel under any non-orthogonal waveform shape. The sparsely subcarrier mapping seems as random subcarriers pattern that was exploited for adaptive equalization with minimum pilot symbols and without need for knowing neither channel sparsity order nor optimum pilot pattern search. It is worth mentioning that the proposed SFDM takes advantages of multicarrier system with reduced number of subcarriers. Spectral efficiency comes from the supported non-orthogonal smooth waveform and reduced GI, along with minimizing pilot symbol rate. Moreover, reducing number of active subcarrier has attractive consequences on PAPR reduction and energy saving per symbol. Based on the observation that, sparser signal can be recovered even under lower SNR ratios, Sparsity adaptation was introduced as alternative strategy instead of redundancy based channel coding. 
Corresponding immunity can be provided through increasing sparsity (by decreasing number of active subcarriers). Finally, the proposed scheme can be adapted to append the modulation group candidates in serving $5 \mathrm{G}$ mobile communications.

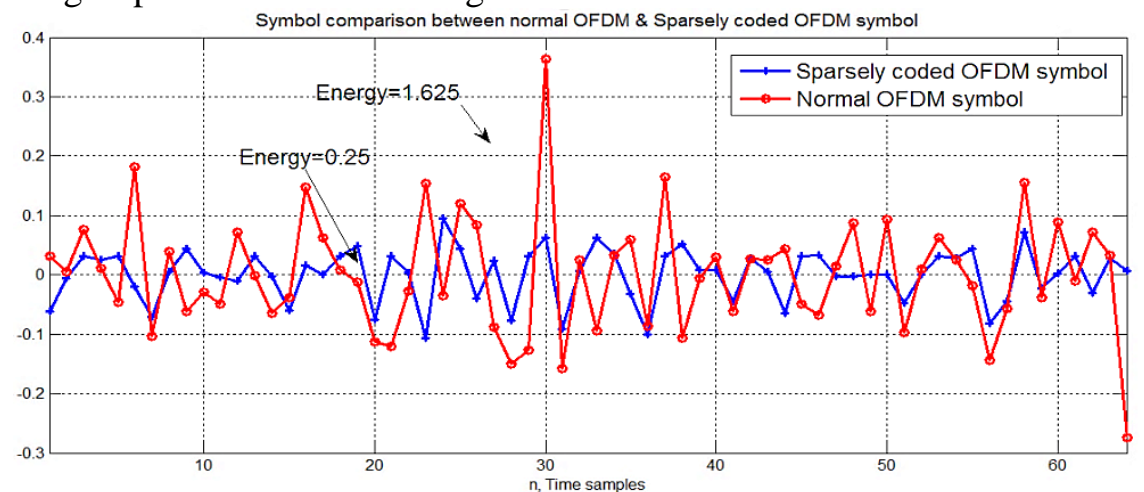

(a)

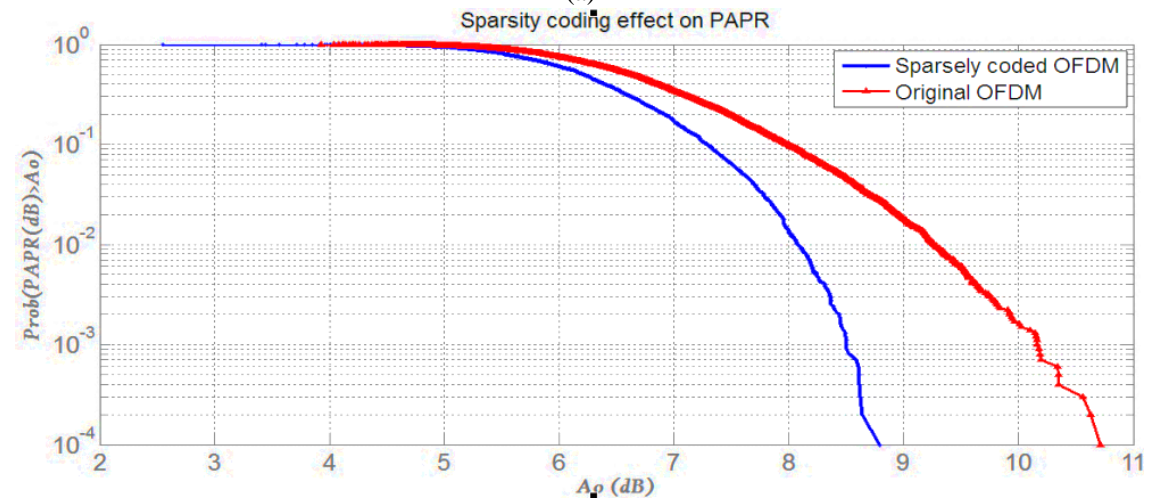

(b)

Fig. 10. (a) sparsely coded OFDM symbol against normal OFDM symbol, (b) Effect of sparsely mapping on PAPR

\section{REFERENCES}

[1] L. Litwin and M. Pugel, "The principles of OFDM, RF Signal Processing" [Last Access July 2016]. Available: http://www.rfdesign.com, pp. 30-48, Jan. 2001.

[2] T. Hwang, C. Yang, G. Wu, S. Li, and G. Y. Li, "OFDM and Its Wireless Applications: A Survey," IEEE Trans. Veh. Technol. IEEE Transactions on Vehicular Technology, vol. 58, no. 4, pp. 1673-1694, 2009.

[3] Elena, L.-Mihaela, I. Madalina, "Considerations regarding the spectral efficiency of orthogonal frequency division multiplexing", 11th International Conference On Development And Application Systems, Suceava, Romania, May 17-19, 2012.

[4] G. Wunder, et. al, "5GNOW: Non-Orthogonal, Asynchronous Waveforms for Future Mobile Applications", IEEE Communications Magazine , Feb. 2014, DOI: 10.1109/MCOM.2014.6736749.

[5] P. Banelli, S. Buzzi, G. Colavolpe, A. Modenini, F. Rusek, and A. Ugolini, "Modulation formats and waveforms for $5 \mathrm{G}$ networks: Who will be the heir of OFDM?," IEEE Sig. Proc. Mag., vol. 31, no. 6, pp. 80-93, Nov. 2014.

[6] A. Farhan, N. Marchetti, F. Figueiredo, and J. P. Miranada, "Massive MIMO and Waveform Design for 5th Generation Wireless Communication Systems,"Proceedings of the 1st International Conference on 5G for Ubiquitous Connectivity, 2014. 
[7] F. Schaich, T. Wild, and Y. Chen, "Waveform contenders for $5 \mathrm{G}$ - suitability for short packet and low latency transmissions", IEEE VTCs, vol. 14, 2014.

[8] Werner Kozek, "Nonorthogonal pulseshapes for multicarrier communications in doubly dispersive channels", IEEE Journal on Selected Areas in Communications , vol. 16 , no. 8 , pp.1579-1589, 1998

[9] A. Sahin, I. Guvenc, and H. Arslan, "A Survey on Multicarrier Communications: Prototype Filters, Lattice Structures, and Implementation Aspects," IEEE Communications Surveys \& Tutorials IEEE Commun. Surv. Tutorials, vol. 16, no. 3, pp. 1312-1338, 2014.

[10] S. W. Smith, The scientist and engineer's guide to digital signal processing. San Diego, CA: California Technical Pub., 2006, (chapter 9).

[11] P. Stoica and R. L. Moses, Spectral analysis of signals. Upper Saddle River, NJ: Pearson/Prentice Hall, 2005.

[12] Y. Hua, A. B. Gershman, and Q. Cheng, High-resolution and robust signal processing. New York: Marcel Dekker, 2004.

[13] M. F. Duarte and R. G. Baraniuk, "Spectral compressive sensing," Applied and Computational Harmonic Analysis, vol. 35, no. 1, pp. 111-129, 2013.

[14] E. Candes and M. Wakin, "An Introduction To Compressive Sampling," IEEE Signal Process. Mag. IEEE Signal Processing Magazine, vol. 25, no. 2, pp. 21-30, 2008.

[15] O. U. Khan, S.-Y. Chen, D. D. Wentzloff, and W. E. Stark, "Impact of Compressed Sensing With Quantization on UWB Receivers With Multipath Channel Estimation," IEEE Journal on Emerging and Selected Topics in Circuits and Systems IEEE J. Emerg. Sel. Topics Circuits Syst., vol. 2, no. 3, pp. 460-469, 2012.

[16] W. U. Bajwa, J. Haupt, A. M. Sayeed, and R. Nowak, "Compressed Channel Sensing: A New Approach to Estimating Sparse Multipath Channels," Proceedings of the IEEE Proc. IEEE, vol. 98, no. 6, pp. 1058-1076, 2010.

[17] Paredes, J.L.; Arce, G.R.; Zhongmin Wang, "Ultra-wideband compressed sensing: channel estimation", IEEE Journal of Selected Topics in Signal Processing, vol 1, no 3, pp. 383 - 395, 2007.

[18] G. Taub" ocka, F. Hlawatscha, and H. Rauhutb, "Compressive estimation of doubly selective channels: exploiting channel sparsity to improve spectral efficiency in multicarrier transmissions”, IEEE Journal of Selected Topics in Signal Processing, 2009.

[19] Eva Lagunas_and Montse N'ajar, "Sparse channel estimation based on compressed sensing for ultra wideband systems", IEEE International Conference on Ultra-Wideband (ICUWB), 2011.

[20] Meng, Jia, Li, Yingying, Nguyen, Nam, Yin, Wotao, Han, Zhu, "High resolution OFDM channel estimation with low speed ADC using compressive sensing", IEEE International Conference on Communications - Kyoto, Japan, 2011.

[21] Wang, Nina, Gui, Guan, Zhang, Zhi, Tang, Tian, Jiang, Jun, "A novel sparse channel estimation method for multipath MIMO-OFDM systems", IEEE Vehicular Technology Conference (VTC Fall) - San Francisco, CA, USA, 2011.

[22] Maechler, Patrick et. Al., "Implementation of greedy algorithms for LTE sparse channel estimation", IEEE, 44th Asilomar Conference on Signals, Systems and Computers - Pacific Grove, CA, USA, 2010.

[23] C. Berger, Z. Wang, J. Huang, and S. Zhou, "Application of compressive sensing to sparse channel estimation,” IEEE Commun. Mag. IEEE Communications Magazine, vol. 48, no. 11, pp. 164-174, 2010.

[24] Haupt, J., Bajwa, W.U., Raz, G., Nowak, R. , “Toeplitz compressed sensing matrices with applications to sparse channel estimation ”, IEEE Transactions on Information Theory, VOL. 56, NO. 11, November 2010 .

[25] N. Wang, Z. Zhang, G. Gui, and J. Jiang, "Improved Compressed Sensing-Based Sparse Channel Estimation Method for Broadband Communication System," Lecture Notes in Electrical Engineering Future Wireless Networks and Information Systems, pp. 465-472, 2012.

[26] P. Pakrooh, A. Amini, and F. Marvasti, "OFDM pilot allocation for sparse channel estimation”, Journal on Advances in Signal Processing, June 2011. arXiv:1106.4507 [cs.IT]. 
Usama S. Mohamed et al., Joint compressive sensing framework for sparse data/channel ...........

[27] L. Applebaum, et.al, "Deterministic pilot sequences for sparse channel estimation in OFDM systems", in 17th International Conference on Digital Signal Processing (DSP), 2011.

[28] C. Qi and L. Wu, "A Study of Deterministic Pilot Allocation for Sparse Channel Estimation in OFDM Systems,” IEEE Communications Letters IEEE Commun. Lett., vol. 16, no. 5, pp. 742-744, 2012.

[29] H. Li, S. Fan, L. Gong, G. Cheng, and S. Li, "Comparisons of Short-Prefix Based Channel Estimation in Single-Carrier Communication Systems," CN Communications and Network, vol. 05, no. 03, pp. 398-402, 2013.

[30] Cheng, Peng, Chen, Zhuo, Gui, Lin, Guo, Y.Jay, Tao, Meixia, Rui, Yun, "Distributed sparse channel estimation for OFDM systems with high mobility", IEEE International Conference on Communications - Budapest, Hungary, June 2013.

[31] Zhang, Y.; Venkatesan, R.; Dobre, O.; Li, C., "Novel compressed sensingbased channel estimation algorithm and near optimal pilot placement scheme", IEEE Transactions on Wireless Communications, Vol. PP, Issue 99, December 2015.

[32] Z. Jellali, L. Najjar, "Tree-based optimized forward scheme for pilot placement in OFDM sparse channel estimation", International Wireless Communications and Mobile Computing Conference (IWCMC), pp. 925 - 929, 2014.

[33] C. Qi, G. Yue, . L. Wu, Y. Huang, and A. Nallanathan, "Pilot design schemes for sparse channel estimation in OFDM systems", IEEE Transactions on Vehicular Technology, VOL. 64, NO. 4, April 2015.

[34] P.Stoica,P.Babu,andJ.Li,"New method of sparse parameter estimation in separable models an $\mathrm{d}$ itsuse for spectral analysis of irregularly sampled data", IEEE Transactions on Signal Processing, VOL. 59, NO. 1, January 2011, pp. $35-47$.

[35] P. Stoica, P. Babu, and Jian Li, "SPICE: a sparse covariance-based estimation method for array processing”, IEEE Transactions On Signal Processing, VOL. 59, NO. 2, February 2011.

[36] Q. Zhang, H. Abeida, M. Xue, W. Rowe and Jian Li, "Fast implementation of sparse iterative covariance-based estimation for array processing", 45 ${ }^{\text {th }}$ Asilomar Conference on Signals, Systems and Computers (ASILOMAR), 2011, pp. 2031 - 2035.

[37] C. R. Rojas, D. Katselis, and H. Hjalmarsson, "A note on the SPICE method", IEEE Transactions on Signal Processing, 2013, Volume: 61, Issue: 18, pp. 4545 - 4551.

[38] P Stoica and P Babu, "SPICE and LIKES: two hyperparameter-free methods for sparseparameter estimation”, Signal Processing, vol 92, 1580-1590, 2012.

[39] P Stoica, D Zachariah and J Li, "Weighted SPICE: a unifying approach for hyper parameterfree sparse estimation", Digital Signal Processing, Vol. 33, pp. 1-12, 2014.

[40] D Zachariah and P Stoica, "Online hyper parameter-free sparse estimation method", IEEE Transactions on Signal Processing", vol. 63, 3348-3359, 2015.

[41] B.L. Sturm - M.G. Christensen, "Comparison of orthogonal matching pursuit implementations", 20th European Signal Processing Conference (EUSIPCO 2012) Bucharest, Romania, August, 2012

[42] W. Dai, O. Milenkovic, "Subspace pursuit for compressive sensing: Closing the gap between performance and complexity", Illinois Univ. at Urbana-Chamapaign, 2008.

[43] S.Sahnoun, P. Comon, A. Pereira da Silva, "A Greedy Sparse Method Suitable for SpectralLine Estimation”, [Research Report] GIPSA-lab, 2016. <hal-01315258>

[44] Miklos Bona, Combinatorics of permutations, CRC Press, Inc., Boca Raton, FL, 2004.

[45] D. L. Kreher and D. R. Stinson. Combinatorial Algorithms: Generation, Enumeration, and Search, chapter 2, pages 42-45. CRC Press, 1 edition, 1999

[46] Knuth, D. E. (2005), "Generating All Combinations and Partitions", The Art of Computer Programming, 4, Fascicle 3, Addison-Wesley, pp. 5-6, ISBN 0-201-85394-9.

[47] M. Salah, Osama A. Omer, Usama S. Mohamed, "Damped frequency division multiplexing: A super-resolution multicarrier scheme", $4^{\text {th }}$ International Japan-Egypt Conference on Electronics, Communications and Computers (JEC-ECC), June 2016.

[48] I. F. Akyildiz and X. Wang, Wireless mesh networks. Chichester, U.K.: Wiley, 2009.

Denis J.G. Mestdagh, "Prior-Art-PAPR-Reduction", 2012, Last Access July 2016: https://www.researchgate.net/publication/281811805 Prior-Art-APRReduction. 


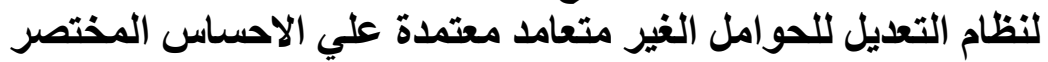

الملخص العربي

يتخذ سلوك القنوات اللاسلكية سلوك متناثر ( sparse) تقريبا في الزمن لهذا فإن طرق الاحساس

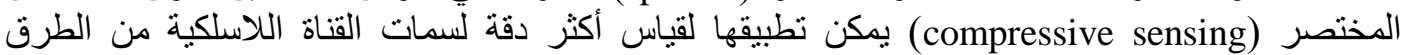

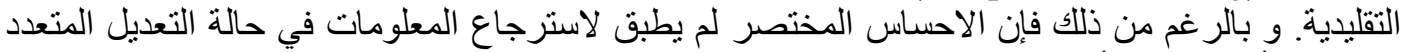

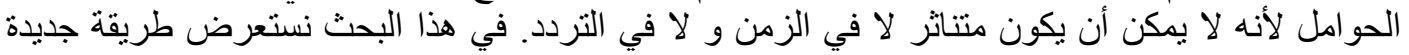

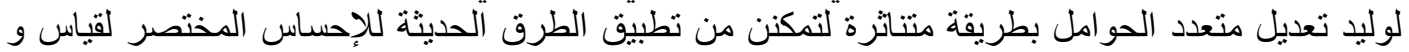

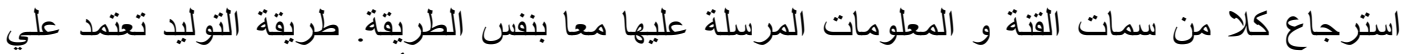

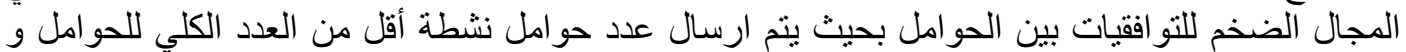

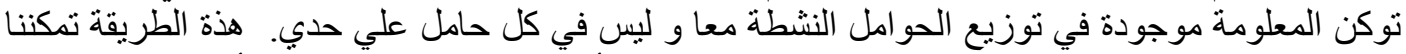

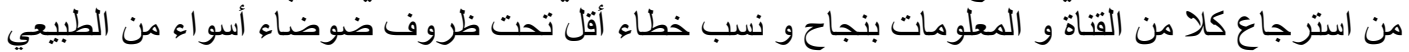

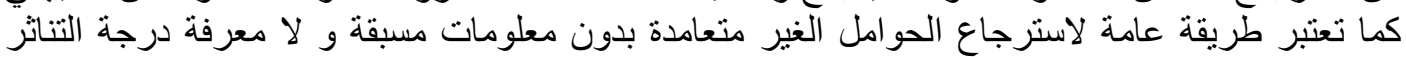

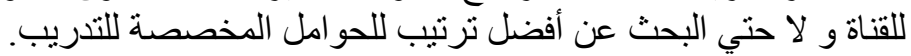

